\section{Predictors of Hemodynamically Successful Stenting for Pulmonary Artery Stenosis - Reply -}

We appreciate Dr Murakami raising the interesting issue of the predictors of hemodynamically successful stenting for pulmonary artery stenosis. His study's data suggested that unilateral branch pulmonary artery stenosis caused compensatory growth of the contralateral pulmonary artery and vascular bed, which may mask the pressure gradient across the stenosis following the shift of blood flow to the contralateral lung. The conclusion was that improvement in the perfusion ratio by stenting for unilateral branch pulmonary artery stenosis can be predicted by the contralateral pulmonary artery diameter and the pressure gradient of the stenosis before stenting. ${ }^{1}$

Unfortunately, we did not have any data for the contralateral pulmonary artery diameter because our study ${ }^{2}$ was based on a retrospective questionnaire survey that collected only the data for the affected side. Consequently, we were unable to evaluate the compensatory growth of the contralateral pulmonary artery.

To evaluate the relationship between hemodynamically effective stenting and the pressure gradient before stenting, we performed 2 subanalyses. First, we extracted 40 lesions that had complete data for the perfusion ratio before and after stenting, as well as the pressure gradient before stenting. Although there may be some selection bias associated with incomplete data, we analyzed the relationship between the percent increase in the perfusion ratio of the affected side to that of the contralateral side ([perfusion ratio of the affected side after stenting/ before stenting] $\times 100$ ), and the pressure gradient of the stenosis before stenting, in this group. There was not a significant correlation between these 2 variables (Figure). Furthermore, we analyzed the relationship between the pressure gradient before stenting and the perfusion ratio after stenting for 32 lesions that had a pre-stenting perfusion ratio less than $30 \%$. Again, there was no significant correlation between these 2 variables (data not shown). From these results, in some of the patients who had a small pressure gradient before stenting, there may be an improvement in the perfusion ratio. In other words, the pressure gradient across the stenosis before stenting alone does not necessarily predict hemodynamically successful stenting. Therefore, we suggest that the result of this evaluation may not directly support the conclusion of Dr Murakami.

Our study has some limitations, including the small number of patients and lack of other hemodynamic data such as right ventricular pressure. Furthermore, there are some differences

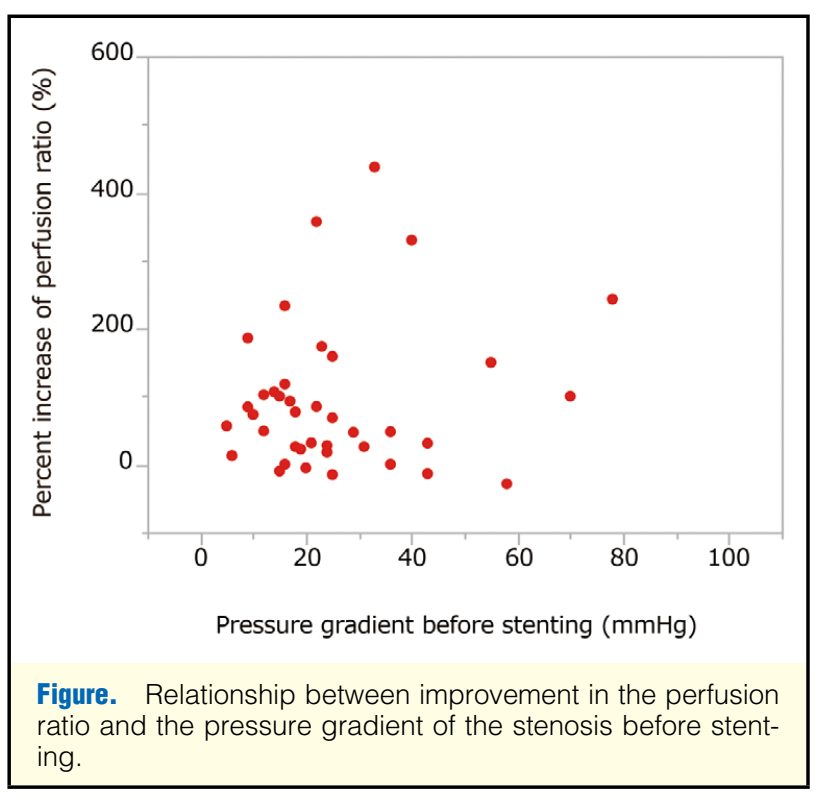

in the laterality of the stenosis (ie, our study included not only left but also other situations such as postoperative pulmonary atresia with/without major aortopulmonary collateral artery and transposition of the great arteries). Underling heart diseases that have been repaired using different surgical methods may also have affected the compensatory mechanism contralateral to the stenosis, whereas Dr Murakami's study included only left pulmonary stenosis in patients following repair of tetralogy of Fallot. We believe further investigation is needed to indicate the predictors of effective stenting.

\section{References}

1. Murakami T, Ueno M. Predictors of hemodynamically successful left pulmonary artery stent implantation in patients after repair of tetralogy of Fallot. J Cardiol 2005; 45: 149-154.

2. Fujii T, Tomita H, Fujimoto K, Otsuki S, Kobayashi T, Ono Y, et al. Morphological and hemodynamic effectiveness of stenting for pulmonary artery stenosis: Subanalysis of JPIC Stent Survey. Circ J 2016; 80: $1852-1856$.

Takanari Fujii, MD

Hideshi Tomita, MD

Cardiovascular Center, Showa University Northern Yokohama Hospital, Yokohama, Japan

(Released online November 5, 2016) 